\title{
Optical far- and near-field femtosecond laser ablation of Si for nanoscale chemical analysis
}

\author{
Vassilia Zorba', Xianglei Mao', Richard E. Russo ${ }^{1,2}$ \\ (1) Lawrence Berkeley National Laboratory, Berkeley, CA 94720, USA \\ (2) Applied Spectra, 46661 Fremond Blvd, Fremont, CA 94538, USA
}

Corresponding author: Dr. Richard E. Russo (RERusso@albl.gov)

\begin{abstract}
Alustract
Extending spatial resolution in laser-based chemical anatysis to the nanoscale becomes increasingly important as nanoscience and nanotechnology develop. Implementation of femtosecond laser pulses arises as a basic strategy for increasing resolution since it is associated with spatially localized material damage. In this work we study femtosecond laser far- and near-field processing of silicon (Si) at two distinct wavelengths ( 400 and $800 \mathrm{~nm}$ ), for nanoscale chemical analysis. By tightly focusing femtosecond laser beams in the far-field we were able to produce sub-micrometer craters. In order to further reduce the crater size, similar experiments were performed in the near-field through sub-wavelength apertures, resulting to the formation of sub-30 $\mathrm{nm}$ craters. Laser Induced Breakdown Spectroscopy (LIBS) was used for chemical analysis with a goal to identify the minimum crater size from which spectral emission could be measured. Enisssion from sub-micrometer craters (full-with-at-half-maximum) was possible, which are among the smallest ever reported for femtosecond LIBS.
\end{abstract}


Keywords: Iaser ablation, LIBS, silicon, far-field, near-field

\section{Introduction}

Lasers are indispensable analytical tools for in siru chemical analysis. [1-4] During the past few years, Laser Induced Breakdown Spectroscopy (LIBS) has emerged as a promising analytical technique which is based on the spectral emisssion from a plasma produced when an intense laser pulse is focused onto a sample surface. Among its advantages is that it can be used for direct and rapid chemical analysis over a wide range of materials and environments including ambient air [5], with little or no sample preparation. One of the main objectives of this technology is to minimize structural damage not only in depth sampling but to also be able to control the lateral crater sizes, which defines the spatial resolution. In most cases, LIBS measurements are from tens to hundreds of micrometers ablation craters, due to difficulty in forming micron order craters as well as measuring emission from a limited quantity of ablated mass.

Femtosecond (fs) lasers have proven to be effective tools in high precision laser-materials processing. Ultrafast lasers offer a number of advantages in materials processing such as high energy flux and limited thermal effects leading to locally confined structural modifications [6]. As a result, their implementation in LIBS has attracted recent interest for reducing material damage. Improving the resolution in fs LIBS is based on tight focusing of pulsed laser irtadiation with high numerical aperture objectives. Additionally, features smaller than the focused beam spotsize can be obtained by adjusting the laser energy threshold for inducing material modification, by taking advartage of the Gaussian pulse spatial distribution [7]. Femtosecond LIBS measurements have been reported from 
craters of $-3 \mu \mathrm{m}$ in diameter in biological samples [8]. Recent results have demonstrated the possibility of measuring LLBS emission from sub-micron craters in thin Cr films [9].

Another strategy for minimizing the laser modified zone to the nanoscale is through non-conventional optical lithrography stucturing schemes which take advantage of near-field effects [10-12]. In far-field laser processing the lateral resolution that can be achieved is restricted by the diffraction limit. For typical laser wavelengths the resolution achieved is on the order of a few hundred nanometers to several microns. However, when a sample is placed in the nearfield of a nanoscale illumination source, the irradiation is physically confined to its immediate vicinity through exploitation of evanescent or non propagating fields. This principle has been implemented in developing apertured near-field scanning optical lithography. In this configuration, laser irradiation incident onto the input of a fiber is guided to its tapered end. This end has an opening (aperture) which typically ranges from a few tens to a few hundred nanometers. Since the size of the focused laser beam is only limited by the size of the aperture in the near-field, one of the main directions in reducing the laser affected sample area is the use of sub-wavelength apertures. The use of apertures in conjunction with fs laser pulses can offer superior spatial resolution in laser sevucturing. One of the main concerns of this technology however is whether enough laser energy for ablation is delivered to the sample through sub-wavelength apertures because of their small transmission efficiency [13]. The majority of studies using subwavelength near-field apertures has been on polymers $[14,15]$ which in prineipal have low ablation thresholds. For chemical analysis however, it is important to not only induce structural modification in a wide range of sample materials [16], but also to ablate enough material for analysis by a suitable detection methods. So far 
the only reports on near-field LIBS were with ns laser induced plasmas $[17,18]$ where emission could be successfully detected from $\sim 2 \mu$ un diameter craters.

In this work, we studied fs laser ablation of Si at 400 and $800 \mathrm{~nm}$ for nanoscale chemical analysis. We compared far- and near-field laser processing and jdentified minimum lateral and axial crater sjze limitations for chemical analysis with LIBS.

\section{Experimental}

A Tì:Sapphire laser (Mai-Tai oscillator coupled to a TSA-25 amplifier, Spectra Physics) was used as the irradiation source, delivering 100 is pulses at a repetition rate of $10 \mathrm{~Hz}$ or $1 \mathrm{~Hz}$. Both the fundamental $(800 \mathrm{~nm})$ and frequency doubled (400 nm) laser wavelengths were used for structuring of single crystalline, intrinsic Si (100) wafers. All measurements were carried out in ambient air at atmospheric pressure and room temperature. Following laser structuring, the samples were scanned by a Nanoscope III Atomic Force Microscope (AFM) system in tapping mode to obtain the surface topographical image. Statistical analysis and line profiles of the surface features were obtained by using the WSXM sottware [19].

\section{Far-field experiments}

For the far-field measurements, laser irradiation was focused on the Si surface by a microscope objective lens (Edmund Optics 20x, 0.60 Numerical Aperture). The light from the induced plasma was collected by a single fiber bundle in close proxinity (a few mm) to the irradiated sample surface. The optical fiber was directly connected with the slit entrance of a spectrometer/ICCD camera system (Princeton Instruments). The spectrometer was equipped with a $600 \mathrm{groove} / \mathrm{mm}$ grating. The focal length of the spectrometer was $150 \mathrm{~mm}$ and the spectral 
resolution was 0.5 nm. The gate of the ICCD samera was triggered by the laser and the relative delay was controlled by the camera. The minimum gate width was 2 ns.

A fresh sample spot was provided by translating an $X Y$ motorized stage before each single laser palse; all data in this study were obtained from single laser pulses (ablation events). Single pulse LIBS experiments were carried out by measuring the spectral entission using a $50 \mathrm{~ns}$ integration time with a delay time of 80 and $60 \mathrm{~ns}$ for the 400 and $800 \mathrm{~nm}$ laser irradiation, respectively. The LIBS emission was measured from the side of the plasma. Each measurement was made on a fresh sample surface, and the average of 10 measurements was calculated. Neutral density filters were used to control the laser pulse energy which was measured by an energy meter (Coherent, J3S-10).

\section{Near-field experiments}

The laser beam ( 400 or $800 \mathrm{~nm}$ ) was coupled to an Near-field Scanning Optical Microscope (NSOM) fiber probe (Vecco) using a $10 \times(\mathrm{NA}=0.25)$ microscope objective lens. The Al-coated tapered end of the NSOM fiber had an aperture of 100-150 nm and was mounted on the head of the NSOM system (Aurora III). The laser energy transmission efficiency through the sub-wavelength aperture system was measured to be $10^{-5}$. Control over the tip-sample distance was achieved via tuning fork shear force feedback, and was kept at g-10 nm [20].

\section{Results and Discnssion}

Far-field processing of Si

Figure 1 (a) shows the far-field LIBS emission from a Si sample in the 195-325 um spectral range as a function of laset energy generated by using a single 400 
$\mathrm{nm}, 100-\mathrm{fs}$ laser pulse irradiation. Strong atomic emission lines of $\mathrm{Si}$ in the UV region are visible; a group of lines around $\sim 252 \mathrm{~nm}(250.6,251.4,251.6,251.9$, 252.4 and $252.8 \mathrm{~nm}$ ) and the characteristic $288.1 \mathrm{~nm}$ line. The AFM surface maps of the ablated \$i surfaces are shown in Figure 1 (b) together with the corresponding lateral profile as a function of laser energy (Figure 1 (c)). The crater size defined as the full-width-at-half-maximum (FWHM) of the feature beneath the original surface can be as low as $1.13 \mu \mathrm{m}$ for the highest energy used and less than $700 \mathrm{~nm}$ for the lowest energy utilized. This value is among the smaillest ever reported for fs LIBS [8,9]. Smaller craters were measured for lower Iaser energies; however no LIBS emission could be detected under these conditions. Indications of peripheral melting/redepositton phenomena are clearly visible through the surface profile plots which show features protruding well above the initial surface in the crater periphery. Even though the height of the surrounding protousions increases with laser energy, the depth of the craters remains fairly stable around $100 \mathrm{~nm}$ independently of the laser energy used.

The LIBS spectra obtained from singlenpulse irradtation of Si using the $800 \mathrm{~nm}, 100 \mathrm{fs}$ laser pulses, are shown in Figure 2 (a). The corresponding AFM images and crater profiles are shown in Figures 2 (b) and (c) respectively. For the lowest lașer energy used, the minimum structure size from which LIBS emission could be measured was - I fum (FWHM). The lateral dimensions increase significantly with laser energy up to $2.3 \mu \mathrm{m}$ in this energy range. The craters formed at this wavelength are deeper compared to the $400 \mathrm{~nm}$ laser itradiation, with their depth remaining over $\sim 180 \mathrm{~mm}$, even for the lower energies used. Similar to the 400 nm laser case, redeposited material is present around the crater; in this case however it does not protrude as much above the initial surface of the samplo material. 


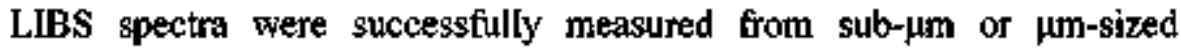
craters, indicating that both wavelengths can be used for spatially confined lateral and axial surface damage. Laser irradiation at $400 \mathrm{~nm}$ apart from leading to the formation of smaller eraters, also results to higher LIBS signal intensities compared to the $800 \mathrm{~nm}$ irradiation, for similar energies (Fignres 1(a) and 1(b)). The emission intensity $I_{p \phi}$ of a transition from a bigher level $p$ to lower level $q$ is expressed as [24]:

$$
I_{p q}=\left(\frac{l}{4 \cdot \pi}\right) \cdot n \cdot\left[\frac{g_{p}}{Z(T)}\right] \cdot A_{p q} \cdot\left(\frac{h \cdot c}{\lambda_{p q}}\right) \cdot \exp \left(\frac{-E_{p g}}{k+T}\right)
$$

where $l$ is the path length of the source, $A_{p q}$ is the transition probability for spontaneous emission, $h$ is the Planck's constant, $c$ is the velocity of light, $\lambda_{p p q}$ is the wavelength of the emission line. $n$ is the total concentration of neutral atom or ion, $g_{p}$ is the statistical weight of the level $p_{1} Z(T)$ is the partition function of atom or ion, $E_{p}$ is the excitation energy of level $p$, and $k$ is the Planck's constant. The temperature governing this energy population is defined as the excitation temperature $T$.

With the rest of the paraneters remain practically the same, the differences in the observed intensities for the two wavelengths used will depend on concentration of neutral atom or ion $n$ and temperature $T$. However, temperature due to its exponential component is expected to affect the line intensity more drastically. The differences in the line intensities observed in each case could thus be correlated to the different temperatures of the formed plasmas, suggesting that higher temperatures are developed in the $400 \mathrm{~nm}$ laser induced plasma.

Qualitatively, the differences in the crater depths for the two laser wavelengths may be assocjated with the optical properties of si for linear absorption at the excitation wavelengths. The optical penetration depths calculated 
through the optical constants of Si [25] for the $400 \mathrm{~nm}$ and $800 \mathrm{~nm}$ irradiation are $164 \mathrm{~nm}$ and $25.5 \mu \mathrm{m}$ respectively. Accordingly, laser irradiation is expected to penetrate deeper into the material bulk for the 800nm laser irradiation case, which is consistent with the deeper craters we observed. It is important however to keep in mind that for the intensities used $\left(\sim 10^{17} \mathrm{~W} / \mathrm{cm}^{2}\right)$ non-linear phenomena (e.g. two-photon absorption) are likely to take place. Additionally, a chemical reaction between the heated/melted Si surface and air can lead to the formation of silicon oxide, chernically modifying the irradiated surface [16]. This process is wavelength dependent and it is in principle favored for lower wavelengths [21]. Finally, the presence of air can aftect the crater formation process for both wavelengths, since the strong recoil pressure exerted on the surface could affect redeposition and resolidification mechanisms [22]. The contribution of these processes to the physical structure and plasma formation mechanisms is under investigation.

Near-field processing of Si

In order to further reduce crater dimensions, we studied near-field laser ablation of $\mathrm{Si}$ through sub-wavelength apertures, using the same wavelengths from the fs laser. Figure 3 (a) shows an AFM surface map of a nano-feature array created using the $400 \mathrm{~nm}$, fs laser irradiation with an NSOM probe output energy of $0.18 \mathrm{~nJ}$. Each row represents regions on the surface processed with a fixed number of pulses (in the range of 5 to 500 ), to demonstrate reproducibility. For a low number of pulses $(<20)$, craters are formed. However tepetitive laser irradiation at this energy causes the craters (Figare 3 (c)) to transform into protrusions (Figure 3 (b)). As a result, craters with a FWHM of $27 \mathrm{~nm}$ to protrusions of $550 \mathrm{~nm}$ (Fignre 3 (b)) could be produced for 1 and 500 pulses 
respectively. Similar structure formation also was observed for slightly higher energies $(0.22 \mathrm{~nJ})[23]$.

When the incident energy was further increased to $0.32 \mathrm{~nJ}$, the structures formed were always craters, independent of the number of laser pulses. Figure 4 (a) shows the structures formed under these conditions for different number of laser pulses. Figure 4(b) shows the craters formed using single-pulse laser irradiation under the same conditions. A FWHM as low as $150 \mathrm{~nm}$ with a depth of $28 \mathrm{~nm}$ was obtained in this case. Formation of more complex patterns (e.g. lines) was also possible by translating the sample with respect to the probe (Figure 4(c)). These measurements demonstrate user control of surface features on the nanoscale by varying laser energy and number of pulses.

Figure $\mathbf{5}$ (a) shows an AFM surface map of a near-field laser processed Si surface using the $800 \mathrm{~nm}, 100 \mathrm{fs}$ laser. Unlike the $400 \mathrm{~nm}$ case, the structures formed are shallow protrusions with a height which remains fairly constant at about $\sim 1 \mathrm{~nm}$ and FWHM lateral sizes ranging from 90 to $700 \mathrm{~nm}$. These structures were formed using 5 to 300 laser pulses respectively. An increase in the laser energy or number of pulses in this case can increase the lateral dimensions up to $-3 \mu \mathrm{m}$, even though their height does not exceed 4 nm after soveral thousand laser pulses (Figure 5 (c)). No structures were observed with single pulse irradiation even under the highest energy used at this wavelength.

The differences described above are representative of the importance of laser wavelength in near-field processing. The mecharisms leading to the formation of these surface structures are complex and interpretation of their role and relative contribution is not an easy task. Qualitatively, in the $400 \mathrm{~nm}$ case a more ablative process may be operative as material appears to be removed from the surface (fonnation of craters). This material may be subsequently redeposited 
on the sample surface, leading to the formation of protrusions. On the other hand, the behavior exhibited for the structures formed using the $800 \mathrm{~nm}$ laser irradiation is more consistent with a surface "melting" mechanism. Again, the differences in the optical penetration depth (single-photon absorption) of si for the $800 \mathrm{~nm}$ itradiation [25] compared to the $400 \mathrm{~nm}$, combined with the differences in the laser energy, suggest that the deposited energy per volume of material will differ significantly [23]. Another factor that should be taken into consideration, as in the far-field laser ablation case, is the influence of the background atmosphere (ambient air) on the formation of these structures, which may affect the formation mechanism and lead to laser induced oxidation of the Si surface. As mentioned previously, this process is favored for lower laser wavelengths [21].

LIBS measurements through the sub-wavelength aperntes for the $400 \mathrm{~nm}$ laser irradiation were unsuccessful. One of the main problems apart from the limited amount of ablated and ionized material was that the optical configuration of the NSOM did not allow for UV omission to be detected, which is the wavelength range where the strong LIBS lines are located. The formation of craters at this wavelength however demonstrates that there is mass removal. On the other hand, it is not clear how easy it would be to get LIBS measurement using the $800 \mathrm{~nm}$ laser since no signs of material ejection were observed with this particular configuration for the range of laser energies tested.

\section{Conclusions}

We demonstrated fs laser ablation of Si using far- and near-field, and studied the effects of laser wavelength ( 400 and $800 \mathrm{~nm}$ ) on surface modifications induced in each ablation scheme. LIBS was an approach to monitor for nanoscale chemical analysis, aiming to identify the minimum crater size from which spectral emission 
could be measured. Dotection of LJBS signal was possible in the far field with sub-nitcrometer resolution, however measurements in the near field under the given experimental conditions were unsuccessful. Detection of LIBS spectral amission in the near field could be possible through improvement of the ablation system and detection scheme. Implementation of larger near-field apertures, as well as proper tuning of the laser parameters (wavelength, pulse duration, energy and number of laser pulses) could lead to the maximization of the ablated mass of a given material, making the detection of LlBS signal easier. Furthermore, optimization of the LIBS spectral emission collection system (collection of light from the induced plasma through high numerical aperture objectives and use of high sensitivity ICCD/spectrograph detector modules) could improve significantly the resolution in LIBS spectral analysis.

Even though there are challenges associated with performing nanoscale chemical analysis in far- and near-field laser processing schemes, in terms of the lateral and axial resolution that can be achieved while maintaining the LIBS spectral emission detection, this is a growing field. The versatility of LIBS as well as minimal sample preparation combined with the ability to operate at ambient air gives the possibility of using this technology for minimal sample damage in different classes of materials. The practical application of interest range from the area of forensics and cultural heritage applications to energy, bio celts and pharmaceutical applications. Finally, the plasmas produced through nanoscale laser ablation.can also be used for spatially resolved quantitative analysis based on mass spectroscopic methods (e.g. Jaser ablation inductively coupled plasma mass spectrometry and secondary ion mass spectrometry). 

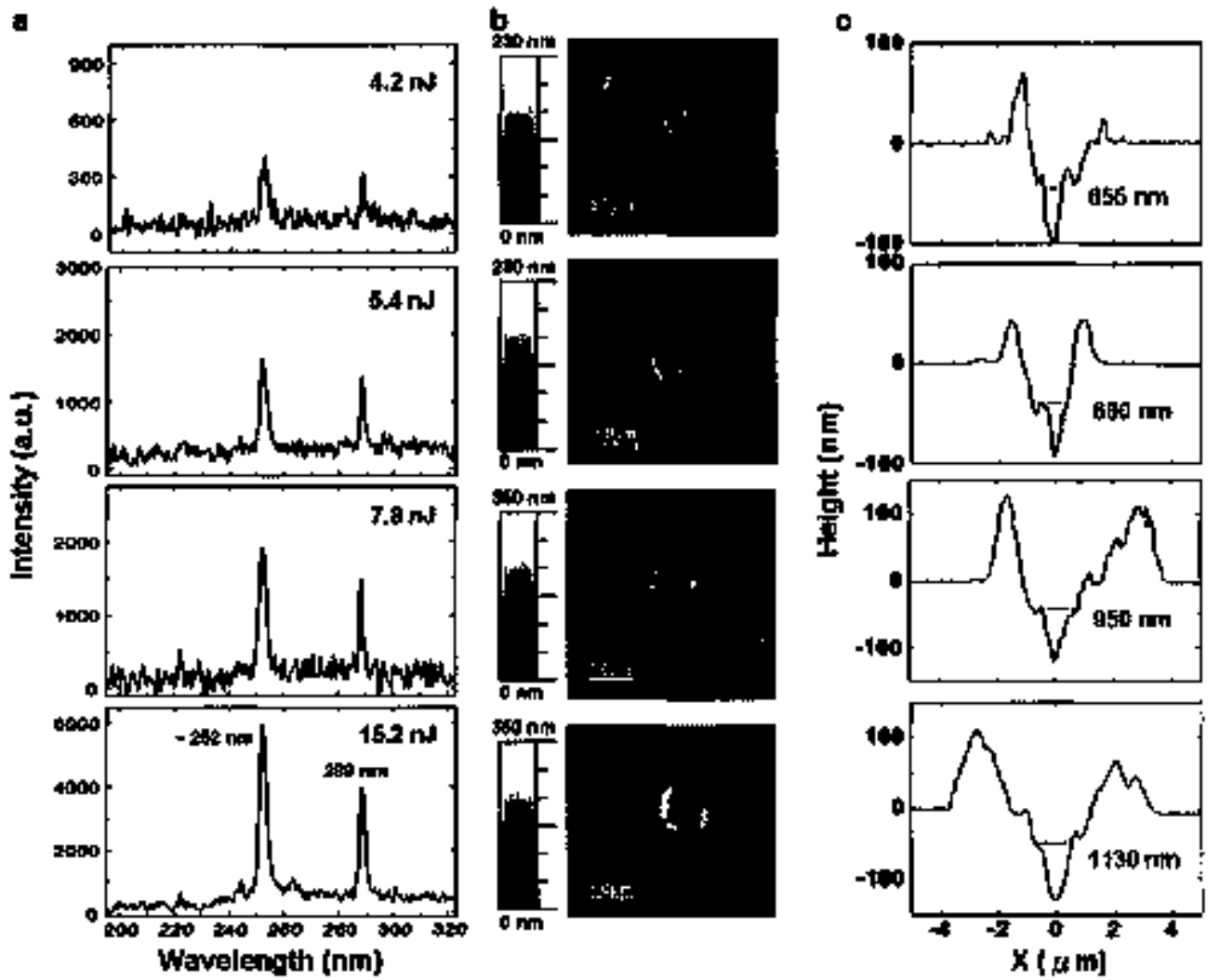

Fig. 1 a LJBS spectral emission generated by a single $400 \mathrm{~nm}$, fs laser pulse, for different pulse energies (4.2-15.2 $\mathrm{nJ}$ ). b AFM surface maps of the corresponding craters and $\mathrm{c}$ their surface profiles 

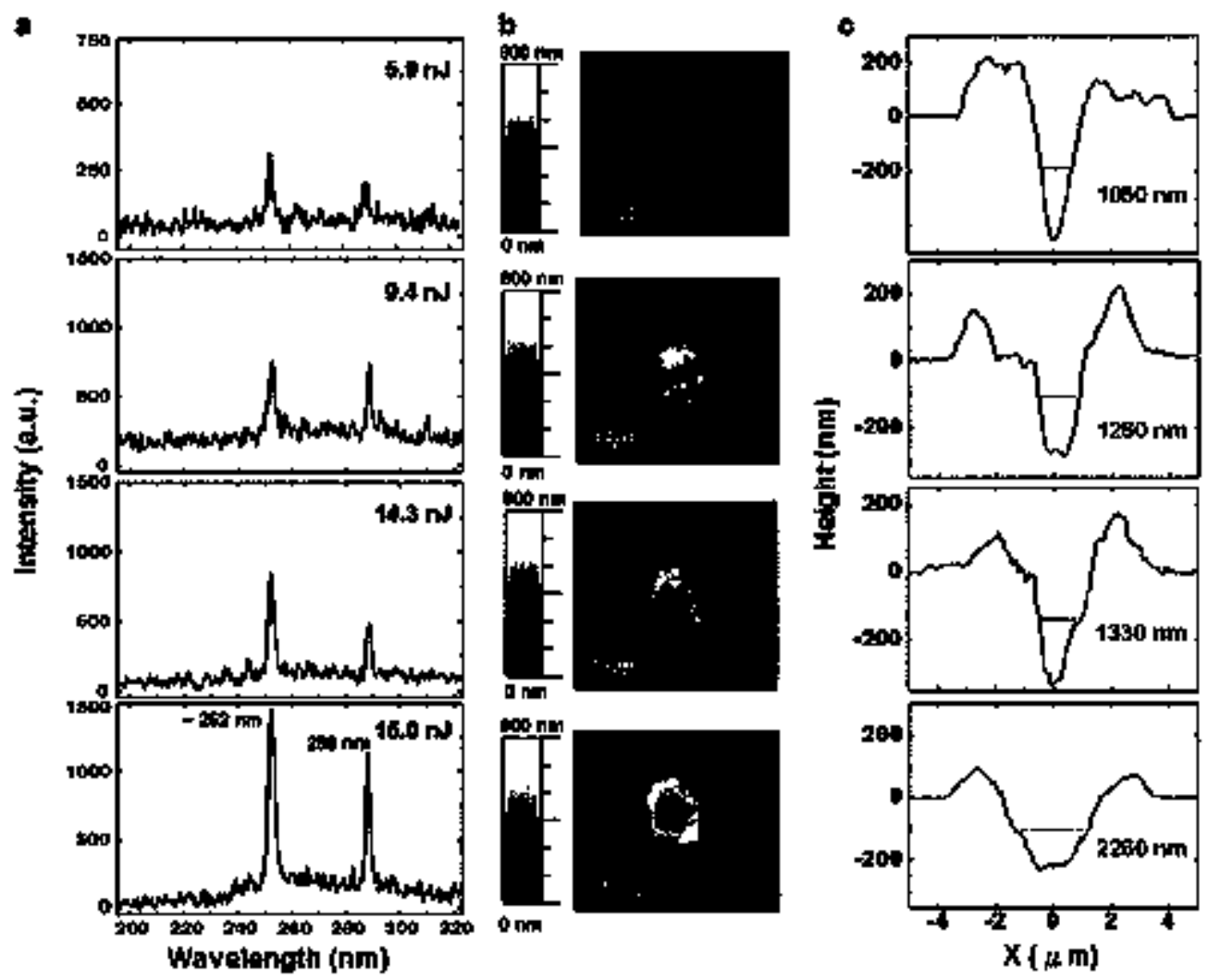

Fig. 2 a LIBS spectral emission generated by a single $800 \mathrm{~nm}$, fs laser pulse, for different pulse energies (5.9-15 $\mathrm{nl}$ ), b AFM surface maps of the corresponding craters and $\mathrm{c}$ their surface profiles 


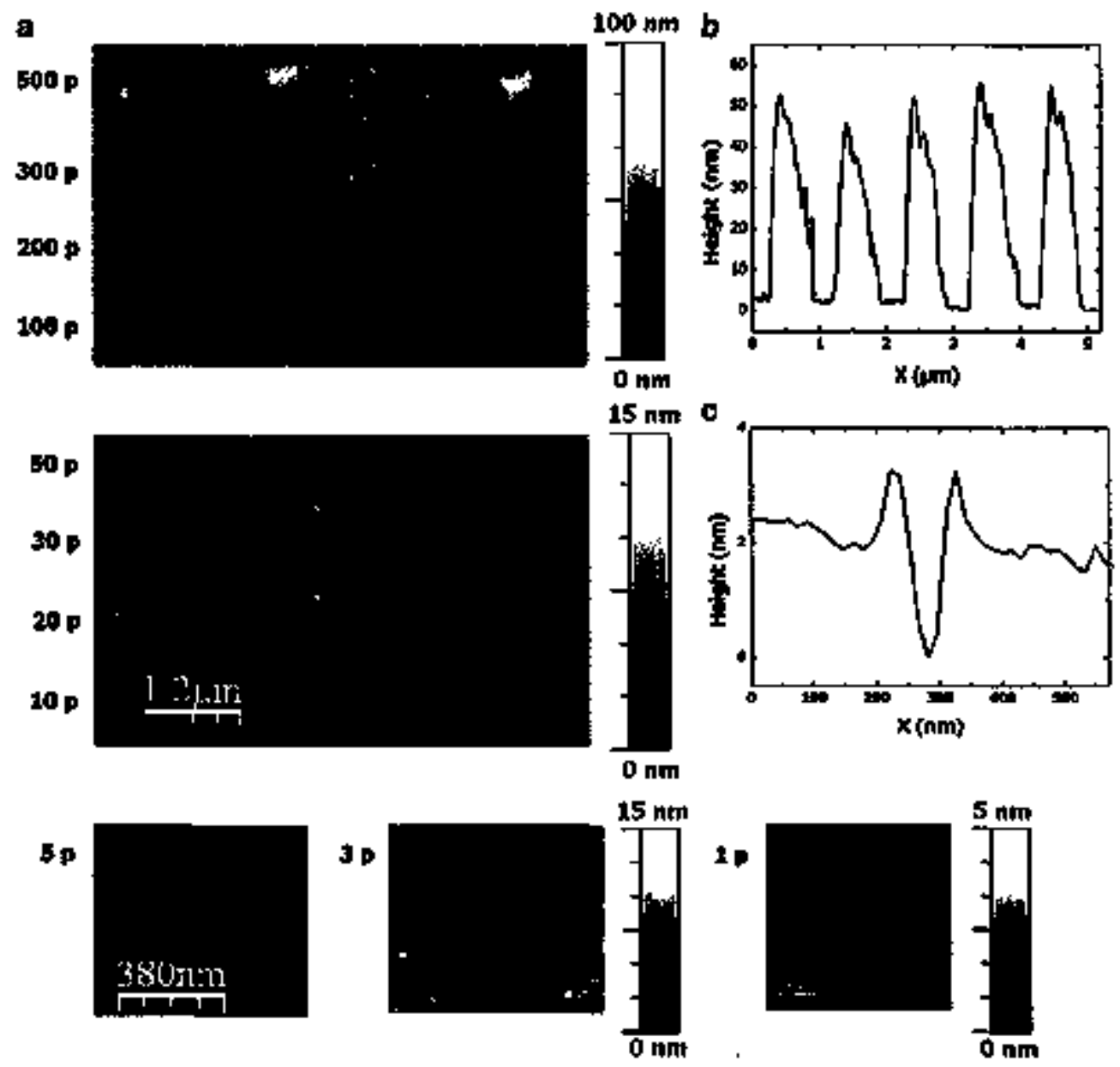

Fig. 3 a AFM surface map of a near-field laser processed $\mathrm{Si}$ surface using the 400 $\mathrm{sm}, 100 \mathrm{fs}$ laser source. Here, ench row represents regions processed with a fixed number of pulses in the range of ten to 500 , at laser energy of $0.18 \mathrm{~nJ}$. The craters formed with $], 3$, and $\$$ pulses are also shown. Surface line profiles for $b 500$ pulses and $\mathrm{e} 10$ pulses 

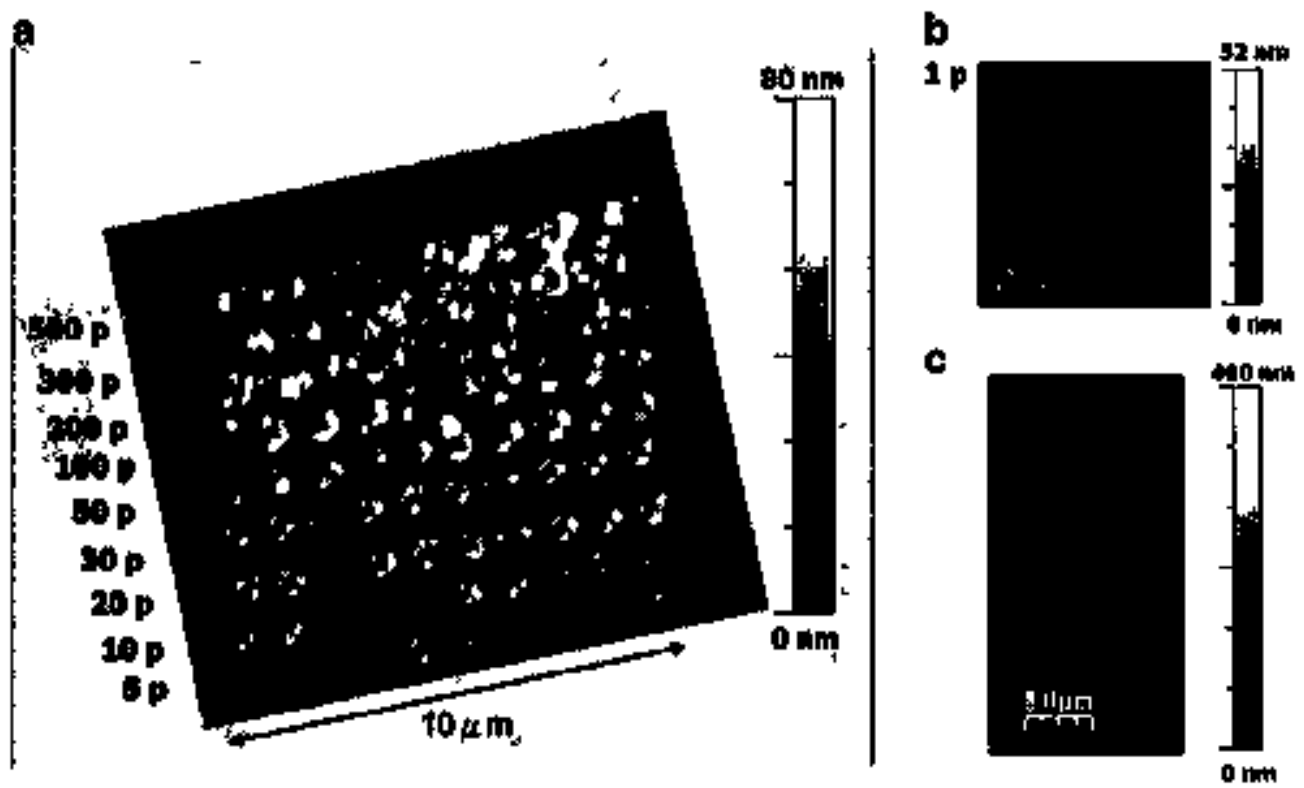

Fyg. 4 a AFM surface map of a near-field laser processed Si surface using the 400 nm, $100 \mathrm{fs}$ laser source. Here, each row represents regions processed with a fixed number of pulses in the range of five to 500 , at laser energy of $0.32 \mathrm{~nJ}$. b AFM image of a crater forned with single pulse irradiation at the same energy. $\mathbf{c}$ Lines formed under these conditions by scaning the sample with respect to the laser beam 

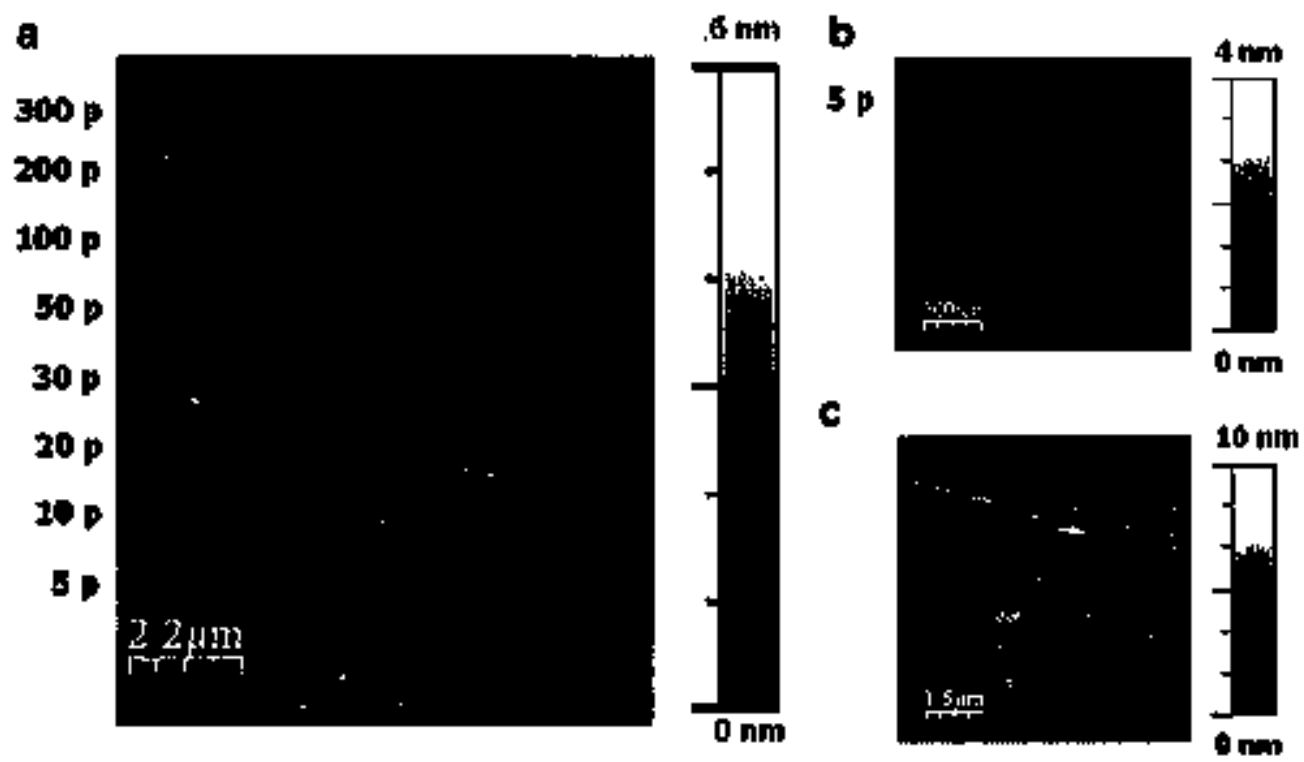

Fig. 5 a AFM surface map of a near-field laser processed \$i surface using the 800 $\mathrm{nm}, 100 \mathrm{fs}$ laser source. Here, each row represents regions processed with a fixed number of pulses in the range of five to 300 , at laser energy of $1.90 \mathrm{~nJ}$. b Higher resolution AFM image of the protrusions formed with five pulses under the same energy. Shallow protrusion formed under 10,000 pulses at $1.27 \mathrm{~nJ}$ 
This research has bees atpported by the Chemical Sclence Division, Office of Basic Energy Sciences, U.S. Department of Energy, under Contract No. DE•AC02-05CHI I23I. The authors afe pleased to acknowsledge helpful discussions with Paul Berdabl af LBNL.

\section{References}

1. Russo RE, Mao XI, Gonzalez JJ, Mao SS (2002) J. Anal. At. Spectrom. 17: 1072-1075.

2. Russo RE, Mao X L, Liu C Gonzalez JJ (2004) J. Anal. At. Spectrom. 19:1084-1089.

3. Durrant SF (1999) J. Anal. At. Spectront. 14: 1385-1403.

4. Gunther D., Jackson SE, Longerich HP (1999) Spectrochim. Acta B 54: 381409.

5. Lee WB, Wu JY, Lee YI, Sneddon J (2004) Applied Spectroscopy Reviews 39: 27-97.

6. Bäuerle D (2000) Laser Processing and Chemistry, Springer, Berlin.

7. Pronko PP, Dutta SK, Squier J, Rudd JV, Du D, Mourou G (1995) Optics Communications 114 : 106-110.

8. Asșion A, Wollenhaupt M, Haag L, Mayorov F, Sarpe-Tudoran C, Winter M, Kutschera U, Baumer T (2003) Applied Physics B-Lasers and Optics 77: 391-397

9. Hwang DJ, Jeon $\mathrm{H}$, Grigoropoulos CP, Yoo J, Russo RE (2007) Applied Physics Letters 91: 251118-1-3.

10. Zenobi $R$, Deckert V (2000) Angew. Chem. Int Ed. 39: 1746* 1756.

11. De Serio M, Zenabi R, Deckert V (2003) Trends Anal. Chem. 22: 70-77. 
12. Kim J, Song KB (2007) Micron 38: 409-426.

13. Zeisel D, Nettesheim S, Dutoit B, Zenobi R (1996) Appl. Phys. Lett. 68: 2491-2492.

14. Lin Y, Hong MH, Wang WJ, Law YZ, Chong TC (2005) Appl. Phys. A 80: $461-465$.

15. Lin $Y$, Hong MH, Wang WJ, Wang ZB, Chen GX, Xie Q, Tan LS, Chong TC (2007) Sensors and Actuators A 133:311-316.

16. Wen SB, Greif R, Russo RE (2007) Applied Physics Letters 91: 251113-1-3.

17. Hwang DJ, Grigoropoulos CP, Yoo J, Russo RE (2006) Applied Physics Letters 89: 254101-1-3.

18. Hwang DJ, Jeon H, Grigoropoulos CP, Yoo J, Russo RE (2008) Joumal of Applied Physics 104: 013110-1-12.

19. Horcas I, Fernandez R, Gomez-Rodriguez JM, Colchero J, Gomez-Herrero J, Baro AM (2007) Review of Scientific Instruments 78:013705-1-8.

20. Betzig E, Trautman JK, Harris TD, Weiner JS, Kostelak RL (1991) Science $251 \div 1468-70$.

21. Schafer SA, Lyon SA (1982) J. Vac. Sci. Tech.: 21, 422-425.

22. Hwang DJ, Grigoropoulos CP, Choi TY. 2006. Journal of Applied Physics 99: 083101-1-5.

23. Zorba V, Mao XL, Russo RE, (2009) Applied Physics Letters 95, 041110-13.

24. Montaser A, Golightly DW, Inductively Conpled Plasmas in Anabytical Atomic Spectroscopy, Second Edition (VCH Publishers, 1992) p.375.

25. Lide DR, CRC Handbook of Chemistry and Physics (CRC Press, Boca Raton, 1994), p. 12-134. 